\title{
RELATIONSHIP BETWEEN THE PERSISTENCE OF THE PROCESSUS VAGINALIS AND AGE IN PATIENTS WITH CRYPTORCHIDISM
}

\author{
LUCIANO A. FAVORITO, WALDEMAR S. COSTA, FRANCISCO J. SAMPAIO
}

\author{
Urogenital Research Unit, State University of Rio de Janeiro, and Division of Urology, Souza Aguiar \\ Municipal Hospital, Rio de Janeiro, Brazil
}

\begin{abstract}
Objectives: To assess if there is an age group where the occurrence of persistent processus vaginalis is more frequent in patients with cryptorchidism.

Materials and Methods: We studied 24 fetuses with gestational age between 23 and 35 weeks postconception (control group) and 102 patients (137 testes) with cryptorchidism aged between 1 and 33 years (mean 10.3 years). We considered 2 situations for analysis of the processus vaginalis: a) complete persistence of processus vaginalis and, b) complete obliteration of the processus vaginalis between the internal inguinal ring and the upper pole of the testis.

Results: Of the 137 cases of cryptorchidism, the processus vaginalis was patent in 79 (57.6\%) and obliterated in $58(42.4 \%)$. Of the 55 patients between 1 and 4 years old, $37(67.2 \%)$ had a patent processus vaginalis and $18(32.8 \%)$ an obliterated one. Of the 37 patients between 5 and 8 years, $17(45.9 \%)$ had patent processus vaginalis and $20(54.1 \%)$ had an obliterated processus. In the 45 patients over 9 years of age, in $25(55.5 \%)$ the processus vaginalis was patent and in 20 $(44.5 \%)$ it was obliterated. In the fetuses, we found 4 cases $(8.3 \%)$ of persistence of the processus vaginalis.

Conclusions: There was no difference in the occurrence of patent processus vaginalis between the various age ranges under study. Patent processus vaginalis was more frequent in patients with cryptorchidism than in fetuses.
\end{abstract}

Key words: testis; cryptorchidism; fetus; congenital abnormalities; surgery; inguinal hernia Int Braz J Urol. 2005; 31: 57-61

\section{INTRODUCTION}

Cryptorchidism is one of the most common congenital pathologies in males, with an incidence ranging from 2 to $5 \%$ of term births, and can reach up to $30 \%$ in premature birth (1-3). Several anomalies are associated with cryptorchidism; however epididymal anomalies and inguinal hernia are among the most frequent (3-5).

The occurrence of inguinal hernias is due to the persistence of the processus vaginalis $(6,7)$. The processus vaginalis is a blind-ending sac extending from the peritoneum to the scrotum, which is layered by celomic epithelium (8). This generally obliterates upon the conclusion of testicular migration $(6,7)$. In cases where the processus vaginalis does not obliterate, the child can develop inguinal hernia or communicating hydrocele (4). When the processus vaginalis is open in patients with cryptorchidism, the incidence of epididymal anomalies is significantly higher than when this structure is obliterated (9-11).

There are several studies in the literature on the incidence of epididymal anomaly and its relation to the persistence of the processus vaginalis (9-11), 
nevertheless, in these studies, patients with pathologies such as hydrocele or inguinal hernia were used as a control group $(10,11)$.

The objective of this work is to study the processus vaginalis in patients with cryptorchidism, observing if there is an age range where the occurrence of persistence of the processus vaginalis is more frequent. As a control group, we used human fetuses whose testes had already completed their migration, which is undiscussed in the literature.

\section{MATERIALS AND METHODS}

From January 2000 to July 2004, we studied 24 fetuses (48 testes) with gestational age between 23 and 35 weeks postconception (WPC), with mean age of 28.7 WPC, and 102 patients (140 testes) with cryptorchidism aged between 1 and 33 years (mean 10.3 years).

All fetuses under study were in a perfect state of conservation with no detectable congenital malformations, with age calculated in WPC by measuring the foot length (12-14). Fetal testes located in the scrotum were used as a control group.

According to their position, cryptorchid testes were classified during surgery into 4 groups: a) abdominal - testis locate above the internal inguinal ring, b) internal inguinal ring - testis in contact with the internal inguinal ring area, c) inguinal canal - testis located between the internal and the external inguinal ring, d) external inguinal ring - testis in contact with the external inguinal ring.

The access used for approaching the processus vaginalis in patients with cryptorchidism was a transversal inguinal incision above the internal in- guinal ring. Once the inguinal canal was opened, the testis and the spermatic vessels were identified and, subsequently, the distal portion of the gubernaculum that united the testis and the scrotum was sectioned, and the spermatic vessels were dissected up to the internal inguinal ring with an incision to the cremaster. Placing a fine forceps inside in order to assess its route assessed the persistence of processus vaginalis. In fetuses, the dissection was performed using as stereoscopic loupe with a magnification of $2.5 \mathrm{x}$.

In order to study the persistence of the processus vaginalis, we considered 2 situations; a) complete persistence of processus vaginalis, and b) complete obliteration of the processus vaginalis between the internal inguinal ring and the upper pole of the testis (Figure-1).

For the contingence analysis of populations under study, we used the qui-square statistical test $(\mathrm{p}<0.05)$ and Fisher's exact test $(\mathrm{p}<0.05)(15)$.

Informed consent was obtained for all cases in this study. The research protocol for this study has been reviewed and approved by our institution's Ethics Committee.

\section{RESULTS}

The positioning of the 140 cryptorchid testes studied and its relation to the persistence of the processus vaginalis is shown in Table- 1 . We observed that most testes $(50.7 \%)$ were located in the inguinal canal. Anorchism occurred in 3 cases $(2.14 \%)$, which were subsequently withdrawn from our sample. Abdominal testes presented the highest index of processus vaginalis persistence (75\%), while the testes lo-

Table 1 - Testicular position in patients with cryptorchidism.

\begin{tabular}{lcc}
\hline Testicular Position & Total $(\boldsymbol{\%})$ & Processus Vaginalis \\
\hline Abdominal & $16(11.4)$ & $12(75)$ \\
Internal Ring & $16(11.4)$ & $10(62.5)$ \\
Canal & $71(50.7)$ & $47(66)$ \\
External Ring & $34(24.2)$ & $10(29)$ \\
Anorchism & $3(2.14)$ & 0 \\
Total & $140(100)$ & $79(57.6)$ \\
\hline
\end{tabular}



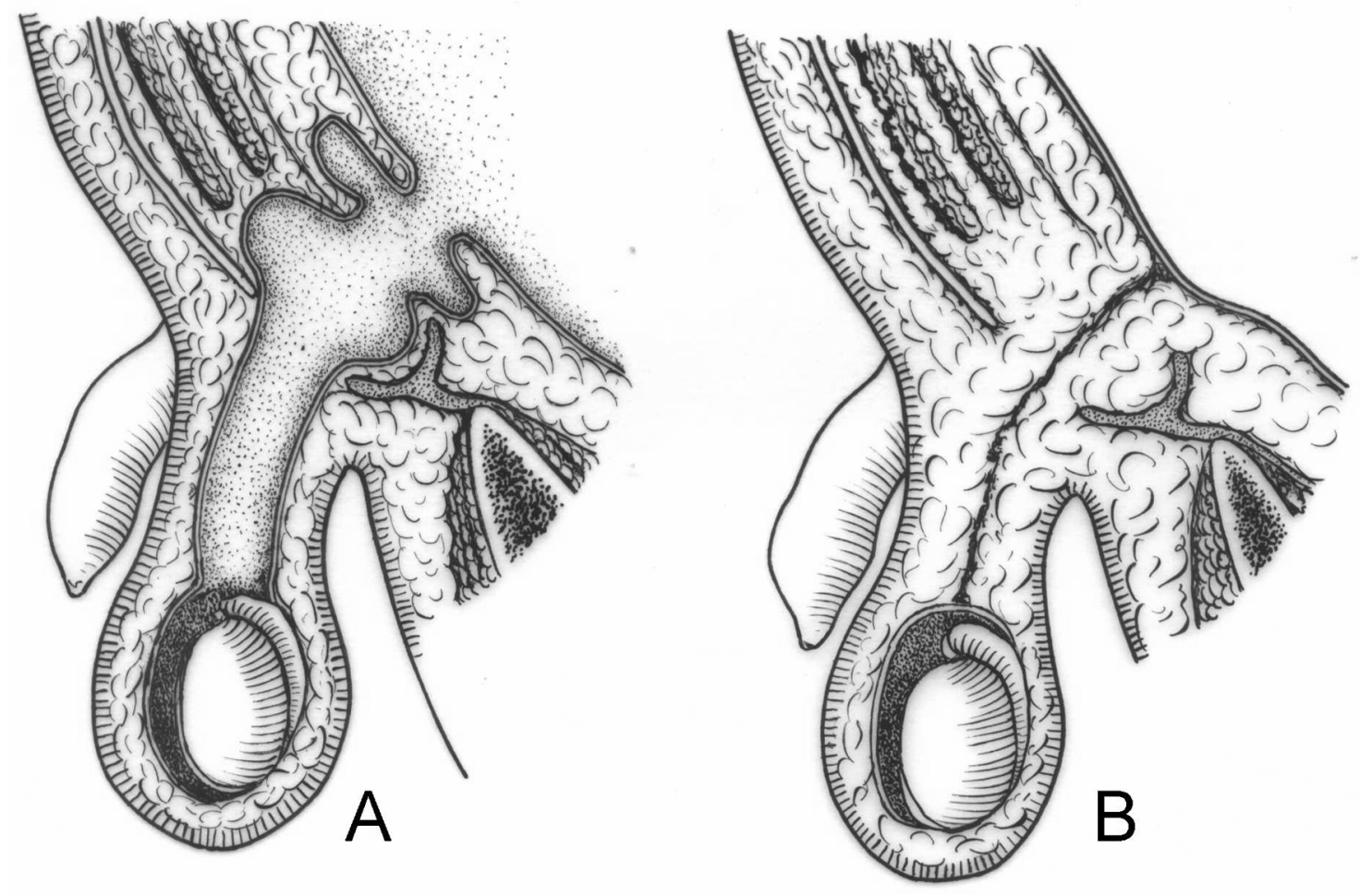

Figure 1 - Schematic drawing demonstrating the persistence of the processus vaginalis (A) and the occluded processus vaginalis (B).

cated in the external inguinal ring presented only $29 \%$ of processus vaginalis persistence.

The relationship between the patient's age and persistence of the processus vaginalis is shown in Table-2. Of the 137 cases under study, the processus vaginalis was patent in $79(57.6 \%)$ and obliterated in $58(42.4 \%)$.

The age range that showed the highest frequency of persistence of processus vaginalis was be- tween 1 and 4 years old (67.2\%). However there was no statistically significant difference between this and the remaining groups; that is, patients between 5 and 8 years of age $(p=0.3749)$ and patients over 9 years of age $(\mathrm{p}=0.2657)$.

In the age range between 1 and 2 years of age, our sample consisted of 24 testes (16 patients), of which $16(66.6 \%)$ presented a patent processus vaginalis.

Table 2 - Persistence of the processus vaginalis in relation to age range. Three patients with anarchism are not computed.

\begin{tabular}{lcc}
\hline AGE & Patent Processus Vaginalis & Obliterated Processus Vaginalis \\
\hline 1 to 4 years $(55 \mathrm{pts})$ & 37 & 18 \\
5 to 8 years $(37 \mathrm{pts})$ & 17 & 20 \\
$>9$ years $(45 \mathrm{pts})$ & 25 & 20 \\
Total = 137 pts & 79 pts $(57.6 \%)$ & $58 \mathrm{pts}(43.4 \%)$ \\
\hline
\end{tabular}


In fetuses whose testes are already positioned in the scrotum, we found 4 cases $(8.3 \%)$ of persistence of the processus vaginalis. When we compared the cases of persistence of the processus vaginalis in fetuses with patients with cryptorchidism, we observed a higher prevalence of persistence of the processus vaginalis in the patients with cryptorchidism $(\mathrm{p}<0.0001)$.

\section{COMMENTS}

The processus vaginalis is a structure identified in all species where there is testicular migration. This conduct allows the passage of the male gonad through the inguinal canal so it can be positioned inside the scrotum, which is the favorable environment for spermatogenesis (6).

Normally, the processus vaginalis obliterates following the testicular migration, separating the tunica vaginalis and the peritoneal cavity. Though it has been frequently postulated that the tunica vaginalis would have one parietal and one visceral layer, some authors state that this is incorrect since the celomic epithelium is absorbed by the tunica albuginea during the embryonal period (16). Thus, the processus vaginalis would form only one parietal layer, which is called tunica vaginalis.

The exact moment when the closing of the processus vaginalis occurs is still unknown. Studies suggest that at birth, there is persistence of the processus vaginalis in up to $80 \%$ of boys, with progressively decreasing rates throughout the first year of life $(7,11,16)$. In a significant number of adult men, the processus vaginalis never obliterates. However, in the majority of these cases, there is no development of indirect inguinal hernia $(7,11,16)$.

There is no agreement concerning the site where the closing of the processus vaginalis begins. According to Scorer \& Farrington (4), the occlusion would progress from the region of the internal inguinal ring to the most distal portion, while Johansen (7) suggests the opposite direction.

The absence of complete regression that leaves a fibrous and non-elastic tissue in the place of the processus vaginalis could be associated with the occurrence of the so-called ascent of the testes. This term is used to describe those testes that, in spite of being initially located in the newborn's scrotum, are identified as cryptorchid at a later age. This vestigial structure would prevent the development of the spermatic cord in response to the somatic growth, anchoring the testis, which would then assume a higher position $(16,17)$.

In our sample, the processus vaginalis was patent in $57.6 \%$ of patients with cryptorchidism and in $8.3 \%$ of fetuses. The incidence of persistence of the processus vaginalis was significantly higher in patients with cryptorchidism than in fetuses whose testes were positioned in the scrotum.

Previous studies have evidenced the persistence of the processus vaginalis in a percentage that is similar to the present study. Jackson et al. (18) identified persistence of vaginal conduct in $51.5 \%$ of cases, and Scorer (19) found a patent conduct in 55\% of cases. Heath et al. (9) detected persistence of the processus vaginalis in only $21.3 \%$ of their sample, while in 64 cases Bica \& Hadziselimovic (20) report persistence of the processus vaginalis in $81.3 \%$ of the cases.

While analyzing patient ages, we observed that in cryptorchidism the age range with the highest frequency of persistence of the processus vaginalis was between 1 and 4 years of age (67.2\%). However, there was no statistically significant difference in relation to the other 2 groups. In the 45 cases where patients were older than 9 years of age, the processus vaginalis was open in $25(55.5 \%)$. These findings do not confirm a trend towards the closing of the processus vaginalis with age.

We conclude that there was no difference in the frequency of patent processus vaginalis between the age ranges under study, and that a patent processus vaginalis was more frequent in patients with cryptorchidism than in fetuses.

\section{REFERENCES}

1. Cendron M, Huff DS, Keating MA, Snyder III HM, Duckett JW: Anatomical, morphological and volumetric analysis: A review of 759 cases of testicular maldescent. J Urol. 1993; 149: 570-3.

2. Kogan S, Hadziselimovic F, Howards SS, Snyder III HM, Huff D: Pediatric Andrology. In: Gillenwater JY, 
Grayhack JT, Howards SS, Snyder III HM (eds.), Adult and Pediatric Urology. St Louis, Mosby. 1996, 3rd ed, vol 3, pp. 2623-74.

3. Gill B, Kogan S: Cryptorchidism. Current concepts. Pediatr Clin North Am. 1997; 44: 1211-27.

4. Scorer CG, Farrington GH: Congenital deformities of the testis and epididymis. London, Butter Worths \& Co, 1971.

5. Marshall FF: Anomalies associated with cryptorchidism. Urol Clin North Am. 1982; 9: 339-47.

6. Shrock P: The processus vaginalis and gubernaculum. Their raison d'etre redefined. Surg Clin North Am. 1971; 51: 1263-8.

7. Johansen TE: Anatomy of the testis and epididymis in cryptorchidism. Andrologia. 1987; 19: 565-9.

8. Backhouse KM: Embryology of testicular descent and maldescent. Urol Clin North Amer. 1982; 9: 315-25.

9. Heath AL, Man DW, Eckstein HB: Epididymal abnormalities associated with maldescent of the testis. J Pediatr Surg. 1984; 19: 47-9.

10. Elder JS: Epididymal anomalies associated with hydrocele/hernia and cryptorchidism: implications regarding testicular descent. J Urol. 1992; 148: 624-6.

11. Barthold JS, Redman JF: Association of epididymal anomalies with patent processus vaginalis in hernia, hydrocele and cryptorchidism. J Urol. 1996; 156: 2054-6.
12. Carr BR, Casey ML: Growth of the adrenal gland of the normal human fetus during early gestation. Early Hum Dev. 1982; 6: 121-4.

13. Hern WM: Correlation of fetal age and measurements between 10 and 26 weeks of gestation. Obstet Gynecol. 1984; 63: 26-32.

14. Platt LD, Medearis AL, DeVore GR, Horenstein JM, Carlson DE, Brar HS: Fetal foot length: relationship to menstrual age and fetal measurements in the second trimester. Obstet Gynecol. 1988; 71: 526-31.

15. Sokol RR, Rohlf FJ: Biometry, 3rd ed. New York, Freeman WH. 1995.

16. Clarnette TD, Hutson JM: The genitofemoral nerve may link testicular inguinoescrotal descent with congenital inguinal hernia. Aust N Z J Surg. 1996; 66: 612-7.

17. Atwell JD: Ascent of the testis: fact or fiction. $\mathrm{Br} \mathrm{J}$ Urol. 1985; 57: 474-7.

18. Jackson MB, Gough MH, Dudley NE: Anatomical findings at orchiopexy. Br J Urol. 1987; 59: 568-71.

19. Scorer CG: The anatomy of testicular descent - Normal and incomplete. Br J Surg. 1962; 49: 357-67.

20. Bica DT, Hadziselimovic F: The behavior of epididymis, processus vaginalis and testicular descent in cryptorchid boys treated with buserelin. Eur J Pediatr. 1993; 152: $38-42$.

\author{
Correspondence address: \\ Dr. Luciano Alves Favorito \\ Urogenital Research Unit \\ Av 28 de Setembro, 87, fundos, térreo \\ 20551-030, Rio de Janeiro, RJ, Brazil \\ Fax: + 5521 2587-6133 \\ E-mail: favorito@uerj.br
}

